\title{
Does insulin-induced increase in the amount of plasma membrane GLUTs quantitatively account for insulin-induced increase in glucose uptake?
}

\author{
K.Zierler \\ Departments of Medicine and Physiology, The Johns Hopkins University School of Medicine, Baltimore, Maryland, USA
}

In 1980 two groups, Cushman and Wardzala [1], and Suzuki and Kono [2], demonstrated independently that insulin causes transfer of glucose transporters (GLUTs) from the interior of suitable cells, in these cases isolated rat adipocytes, to the plasma membrane. The original laboratories have confirmed their conclusion repeatedly, as have a number of others. The general conclusion, that insulin increases the GLUT content of plasma membranes of cells that respond to insulin with increased glucose uptake, has also been supported by observations, not only in true adipocytes, but also in 3T3-L1 adipocyte-like cells [3-5], in rodent brown fat [6], in rat skeletal muscle [7-10], and in rat heart muscle [11, 12].

There is now no doubt that, in those cells that respond to insulin with increased glucose uptake, at least one effect of insulin that leads to increased glucose uptake is enrichment of plasma membrane GLUT by recruitment from the cell's interior. The question is, does insulin-induced increase in plasma membrane GLUT density completely account for insulin-stimulated glucose uptake?

The purpose of this article is to analyse published data in which insulin effects on glucose uptake and on increase in the number of the two glucose transporter isoforms GLUT1 and GLUT4, in the cell plasma membrane are reported, to see if they support the claim that the only, or by far the predominant, effect of insulin on glucose uptake is translocation of glucose transporters, GLUTs, from the cell's interior to its plasma membrane or support an alternative hypothesis, that a substantial portion of insulin-induced glucose uptake is due to alteration of the intrinsic ac-

Corresponding author: K.Zierler, M.D., Departments of Medicine and Physiology, Johns Hopkins University School of Medicine, 600 N. Wolfe Street/Blalock 904, Baltimore, MD 21287-4904, USA

Abbreviations: GLUT, Glucose transporter. tivity of a GLUT, such that the rate at which a GLUT transports glucose is increased. Both hypotheses have been offered many times, and both are approximately as old as the original reports of GLUT translocation. It is not my purpose to review the various points of view, or the reasons advanced by proponents of one view or another, but simply to examine pertinent reported data in the light of an essentially model-free analysis.

Although absolute values of D-glucose uptake, or of its analogues, 2-deoxy-D-glucose and 3- $O$-methyl-D-glucose, are measured, most reports do not present absolute values of the GLUT isoforms. For this reason, although investigators have confidence in the observed value of the ratio of densities of GLUT1, and that of GLUT4, in the plasma membrane in the presence and absence of insulin, there is less confidence in the ratio of GLUT1 to GLUT4, or in the absolute value of either, except in those few reports in which assays of the two GLUTs have permitted quantitative comparison between (GT1) and (GT4), the density or concentration of the two GLUTs in the plasma membrane.

It is an experimental observation, in those reports in which all three ratios were measured, that the ratios of values with insulin to those without insulin stand in the following order: glucose uptake ratio > plasma membrane GLUT4 ratio > plasma membrane GLUT1 ratio (see Table 1 for references). Some investigators have used this inequality to mean (i) that translocation of GLUT to the plasma membrane does not entirely account for insulininduced glucose uptake and that one must therefore hypothesize that insulin increased GLUT intrinsic activity. Others conjecture that (ii) past technical problems, which progress has tended to overcome mainly by use of impermeant markers of cell surface GLUT, were responsible for the discrepancy, and that, because the ratio of GLUT4 seemed to be ap- 
Table 1. Glucose uptake and GLUT density ratios

\begin{tabular}{lcccl}
\hline Cell type & $Q_{i} / Q_{b}$ & $G T 4_{i} / G T 4_{b}$ & $\mathrm{GT}_{\mathrm{i}} / G T 1_{b}$ & Reference \\
\hline 3T3-L1 & 9.5 & 2.8 & 1.6 & 14 \\
3T3-L1 & 21.0 & 17.0 & 6.5 & 15 \\
3T3-L1 & 18.6 & 11.3 & 3.4 & 13 \\
Adipocyte & 9.1 & 4.9 & 2.5 & 16 \\
Adipocyte & 55.0 & 16.0 & 9.5 & 17 \\
Adipocyte & 30.0 & 14.8 & 10.7 & 17 \\
Adipocyte & 23.4 & 18.3 & 4.6 & 18 \\
Adipocyte & 5.2 or 11.0 & 2.7 & 0.9 & 19 \\
Muscle $^{\mathrm{b}}$ & 3.1 & 1.5 & 1.0 & 20 \\
Muscle & 2.2 & 1.6 & 1.0 & 21 \\
Muscle $^{\mathrm{d}}$ & 3.6 & 2.5 & 1.0 & 21 \\
Muscle $^{\mathrm{e}}$ & 3.9 & 1.4 & 1.0 & 22 \\
Muscle $^{\mathrm{f}}$ & $8-13$ & 1.8 & - & 23 \\
Muscle $^{\mathrm{g}}$ & 3.5 & 1.6 & - & 24 \\
Muscle $^{\mathrm{h}}$ & 8.1 & 4.0 & - & 25 \\
Muscle $^{\mathrm{i}}$ & 10.6 & 2.5 & - & 25 \\
Muscle $^{\mathrm{j}}$ & 5.0 & 2.6 & 1.0 & 26 \\
Muscle $^{\mathrm{k}}$ & $7-8.5$ & 7 & - & 27 \\
\hline Alladipocyte & & $7 \mathrm{n}$ & &
\end{tabular}

All adipocyte and muscle preparations are from rat unless stated otherwise. ${ }^{\text {a }}$ Lower value of $Q_{i} / Q_{b}$ with $4 \mu \mathrm{mol} / 12$-deoxy-Dglucose; higher value with $20 \mu \mathrm{mol} / 1$ glucose. Glucose concentration for GLUT assay not reported. ${ }^{\mathrm{b}}$ Plasma membranes (PM) from mixed forelimb muscles. ${ }^{\mathrm{c}} \mathrm{PM}$ from red gastrocnemius. ${ }^{\mathrm{d}} \mathrm{PM}$ from white gastrocnemius. ${ }^{\mathrm{e}} \mathrm{PM}$ from mixed soleus, red and white gastrocnemius from Zucker lean rats. ${ }^{\mathrm{f}}$ Range of glucose uptake values, ascending order, for white gastrocnemius, plantaris, red gastroc and soleus, obtained by hindlimb perfusion; GLUT value obtained on PM of mixed hindlimb muscles. ${ }^{\mathrm{g}}$ Biopsy of vastus lateralis in normal controls (for study of NIDDM); glucose uptake by muscle strips; GLUT assayed in PM. ${ }^{\mathrm{h}}$ Glucose uptake measured by 3-O$\left[{ }^{3} \mathrm{H}\right]$ methyl glucose content of excised triceps surae following hindlimb perfusion in the mouse; GLUT assayed in PM from

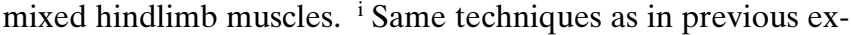
periment but in transgenic mice overexpressing GLUT4. ${ }^{\mathrm{j}}$ Glucose uptake by perfused hindlimb muscles; GLUT assayed in mixed muscle PM. ${ }^{k}$ Glucose uptake ratios varied over the indicated range in four sets of experiments on excised whole soleus muscles; GLUT assayed in PM

proaching that of glucose uptake (and in a few cases equaling it, although not in reports in which GLUT1 was also measured), translocation of GLUT4 to the plasma membrane accounts for all, or nearly all insulin-induced increase in glucose uptake. The assumption, which turns out to be wrong, in this conjecture is that all that is necessary is that the fold increase in plasma membrane GLUT4 be as great as that of glucose uptake. Others suggest (iii) that the discrepancy between insulin-induced fold increase in glucose uptake and that of plasma membrane GLUT4 might be accounted for if the intrinsic activity of GLUT4 exceeds that of GLUT1, and that the insulin-induced increase in plasma membrane GLUT4 might, in this way, completely account for the increased glucose uptake. My purpose is to test these three conjectures. The analysis demonstrates that if published data are reliable for purposes of this analysis, conjectures (ii) and (iii) must be rejected.
The strategy of my analysis is: (a) to set up a modelfree relationship between glucose uptake and cell plasma membrane GLUT1 and GLUT4 densities which includes the case in which insulin increases the intrinsic activity of one or both GLUT isoforms, and which assumes only that glucose transport in the tissues examined is mediated only by GLUT1 and GLUT4, (b) then to assume that insulin makes no change in GLUT intrinsic activity. If this assumption makes it possible for the increase in plasma membrane GLUT density to account completely for observed glucose uptake, then the question is answered; thus, it would not be necessary to propose that there is an additional effect of insulin to increase glucose uptake. On the other hand if the analysis reveals a discrepancy between observed glucose uptake and that portion of it that can be attributed to recruitment of GLUT to the plasma membrane, the answer to the question is, with a caveat noted later, that some additional effect of insulin is required, and we may be able to state quantitatively how big a role recruitment plays.

\section{Definitions, syntax, and equations}

The rate $Q$, at which glucose is transferred across a cell membrane by any given GLUT isoform is dissected as the product of some average number $r$, of molecules of glucose transported per unit time per molecule of the GLUT isoform and the number of molecules of the isoform $(G T)$, per cell surface (or other reference base):

$$
Q=r(G T)
$$

This is a statement of syntax, a definition of $r$. As such, it can be neither true nor false. (An example of statements of syntax: we agree that it is useful to construct a sequence of numbers we shall call "counting" numbers. For example, we agree that the counting number we shall call 3 is constructed by adding the number 1 to the number 2.) No assumptions are made about processes underlying the content of any of the three variables in eq(1). This, then, is a model-free analysis.

The factor $r$ has dimensions of $1 /$ time; that is, it has dimensions of a rate coefficient, and it has the meaning of turnover number, as that expression is used in enzymology. It is a coefficient, not necessarily a constant. Although we are not concerned here with its possible internal structure, we know that $r$ is at least a function of D-glucose concentration, because glucose uptake is a saturable process. We accept that $r$ is an average rate coefficient because, in a given population of a specified GLUT isoform, it is not required that every GLUT be transporting glucose at the same rate. I will use the term "intrinsic activity" interchangeably with "turnover number". (Although others have used these two terms just as I am using them, still others 
have used them to mean something different.) Note again that no mechanism by which GLUT transports glucose is implied in the factor $r$ or in eq(1).

Our interest is in those cell types that respond to insulin by increasing glucose uptake and that have two GLUT isoforms GLUT1 and GLUT4. With respect to the contribution to observed glucose uptake made by translocation via GLUT1, eq(1) takes the form $Q 1=r 1(G T 1)$. Similarly, the contribution made by GLUT4 is $Q 4=r 4(G T 4)$. If GLUT1 and GLUT4 are the only GLUT isoforms in the cell under study, then the experimentally observed glucose uptake $Q$, is the sum of (unmeasured) glucose uptake via GLUT1 and via GLUT4: $Q=r 1(G T 1)+r 4(G T 4)$. This simply states that we require that there be no way in which $D$-glucose can be transported into the cells under study except via GLUT1 and GLUT4.

Now consider the components of observed glucose uptake during the basal state, indicated by subscript $b$, and during acute response to insulin, indicated by subscript $i$. This produces the pair of equations

$Q_{b}=r 1_{b}(G T 1)_{b}+r 4_{b}(G T 4)_{b}$.

$Q_{i}=r 1_{i}(G L 1)_{i}+r 4_{i}(G T 4)_{i}$.

which has the 4 unknown $r$ s, whose value, therefore, cannot be determined uniquely.

There are several ways to proceed from this point.

(a) When we make the assumption that insulin has no effect on the turnover number, we reduce the number of unknowns to two: $r 1 \equiv r 1_{b}=\mathrm{r} 1_{\mathrm{i}}$ and $r 4 \equiv r 4_{b}=\mathrm{r} 4_{\mathrm{i}}$. The pair of simultaneous linear equations (2) can now be solved explicitly for $r 1$ and $r 4$ by the usual methods. When we plug in numerical values from the literature, we find that either $r l$ or $r 4<0$, or $r 1=0$. Because these are inadmissible results (the $r$ s must be greater than 0 ), the assumption that insulin has no effect on turnover number must be rejected.

This analysis, although stating that translocation is not the whole story, does not give us an idea of what fraction of insulin-induced glucose uptake translocation might account for.

(b) Divide eq(2b) by eq(2a), right hand side by right hand side and left hand side by left hand side, and rearrange to express insulin effects in terms of ratios so that we can make use of reported data:

$$
\begin{aligned}
& \frac{r 4_{i}(G T 4)_{i}}{r 4_{b}(G T 4)_{b}}-\frac{Q_{i}}{Q_{b}}= \\
& \left(\frac{Q_{i}}{Q_{b}}-\frac{r 1_{i}(G T 1)_{i}}{r 1_{b}(G T 1)_{b}}\right) \frac{r 1_{b}(G T 1)_{b}}{r 4_{b}(G T 4)_{b}}
\end{aligned}
$$

We now assume that insulin has no effect on the turnover numbers. $\mathrm{Eq}(3)$ now simplifies to
$\frac{(G T 4)_{i}}{(G T 4)_{b}}-\frac{Q_{i}}{Q_{b}}=\left(\frac{Q_{i}}{Q_{b}}-\frac{(G T 1)_{i}}{(G T 1)_{b}}\right) \frac{(G T 1)_{b}}{a(G T 4)_{b}}$

where $a \equiv r 4 / r 1$. Both sides of eq(4) must be positive or both sides must be negative. It is a consistent observation in all reports that $\left(Q_{i} / Q_{b}\right)>(G T 1)_{i} /$ $(G T 1)_{b}$. (Table 1). Therefore, with that knowledge and from eq(4) it follows that if the underlying assumptions are correct there must then be the following sequence of inequalities:

$\frac{(G T 4)_{i}}{(G T 4)_{b}}>\frac{Q_{i}}{Q_{b}}>\frac{(G T 1)_{i}}{(G T 1)_{b}}$

Inequality (5) states that so long as $\left(Q_{i} / Q_{b}\right)>(G T 1)_{i} l$ $(G T 1)_{b},(G T 4)_{i} /(G T 4)_{b}$ must be greater than $\left(Q_{i} / Q_{b}\right)$ if the assumptions are correct. It is not even enough for $(G T 4)_{i} /(G T 4)_{b}$ to equal $\left(Q_{i} / Q_{b}\right)$. From eq(4) if the assumptions are correct the only condition under which it can be observed that $(G T 4)_{i} /(G T 4)_{b}=\left(Q_{i} /\right.$ $\left.Q_{b}\right)$ is one in which $\left(Q_{i} / Q_{b}\right)=(G T 1)_{i} /(G T 1)_{b}$. Because that has never been the case, even if $(G T 4)_{i} l$ $(G T 4)_{b}=\left(Q_{i} / Q_{b}\right)$, one or more of the assumptions must be rejected.

There are a few reports from which one can calculate the size of the ratio " $(G T 4)_{i} l(G T 4)_{b}$ " that is required if insulin has no effect on turnover number, and the ratio " $\left(Q_{i} / \mathrm{Q}_{\mathrm{b}}\right)$ ", which is that part of the observed $\left(Q_{i} / Q_{b}\right)$ that can be ascribed only to translocation of GLUT to the plasma membrane. It is the value we should have found for $\left(Q_{i} / Q_{b}\right)$ if there had been no increase in GLUT intrinsic activity.

Rearrange eq(4) to make explicit the fold increase in (GT4) required if the observed insulin-induced increase in glucose uptake is to be accounted for entirely by GLUT recruitment to the plasma membrane:

“( $\left.\frac{G T 4_{i}}{G T 4_{b}}\right) ”=\frac{Q_{i}}{Q_{b}}+\left(\frac{Q_{i}}{Q_{b}}-\frac{(G T 1)_{i}}{(G T 1)_{b}}\right)\left(\frac{\left(G T 1_{b}\right.}{a(G T)_{b}}\right)$

Another rearrangement of eq(4) makes explicit the ratio of glucose uptake with and without insulin that can be ascribed purely to increased plasma membrane GLUT:

“( $\left.\frac{Q_{i}}{Q_{b}}\right) ”=\frac{(G T 1)_{i}+a(G T 4)_{i}}{(G T 1)_{b}+a(G T 4)_{b}}$

Values for glucose uptake ratios and the various GLUT densities and their ratios appear in Table 2. On the right-hand side of eqs (6) and (7) there remains the as yet unknown parameter $a$, the ratio $r 4_{b}$ l $r l_{b}$. There are three reports that supply data from which the ratio $a$ can be calculated. From data of Palfreyman et al. [13] on 3T3-L1 adipose-like cells, in their Table 1, for basal state, $r l_{b}=2.4 \times 10^{4} \mathrm{~min}^{-1}$, $\mathrm{r}_{\mathrm{b}}=4.0$, whence $a=1.7$. Data presented are inadequate to determine whether this value differs signifi- 
Table 2. Percent increase in glucose uptake accounted for only by translocation

\begin{tabular}{|c|c|c|c|c|c|c|c|}
\hline Cell type & $G T 4_{i} / G T 1_{i}$ & $G T 4_{b} / G T 1_{b}$ & "GT4 $/ G T 4_{b}$ " & $" G T 4_{i} / G T 4_{i} "$ & $" Q_{i} / Q_{b} "$ & $\% " Q_{i} " / Q_{i}$ & Refs \\
\hline 3T3-L1 & 0.66 & 0.30 & 71 & 4.2 & 8.3 & 40 & 15 \\
\hline 3T3-L1 & 0.89 & 0.27 & 74 & 6.6 & 3.4 & 27 & 13 \\
\hline Adipose & 8.1 & 2.0 & 33 & 1.8 & 13.8 & 59 & 18 \\
\hline Adipose & 2.7 & 0.92 & 20 & 7.3 & 1.9 & 17 & $19^{\mathrm{a}}$ \\
\hline Adipose & 2.7 & 0.92 & 9 & 3.3 & 1.9 & 36 & $19^{\mathrm{a}}$ \\
\hline Muscle & 9.0 & 6.30 & 4 & 3.1 & 1.4 & 35 & $22^{\mathrm{b}}$ \\
\hline
\end{tabular}

"GT4 ${ }_{i} / G T 4_{b}$ ", the ratio necessary if insulin-induced translocation of GLUT4 to the plasma membrane were to account entirely for the observed increased glucose uptake. "GT4 $4_{i}$ " $G T 4_{i}$, the factor by which observed $G T 4_{i}$ must be multiplied if insulin-induced translocation of GLUT4 to the plasma membrane were to account entirely for the observed increased glucose uptake. " $Q_{i} / Q_{b}$ ", the fold increase in glucose uptake that would have been observed if translocation of GLUT1 and

cantly from 1 . Nishimura et al. [28], in experiments with oocytes overexpressing GLUT1 and GLUT4, calculate $r 1_{b}=2.7 \times 10^{4} \mathrm{~min}^{-1}$, and $r 4_{b}=1.7$, whence $a=0.63$, which is not statistically significantly different from 1. The third report [29], also on Xenopus oocytes transfected to overexpress GLUTs, supplies only the ratio $r 4_{b} / r 1_{b}, a=0.45$, which was significantly less than 1. It is difficult to know how much confidence to place in any of the reported values for turnover number of a specified GLUT isoform because calculations have been made on the basis of ratios of calculations remote from the primary data (assumption of Michaelis-Menten kinetics), and under decidedly artificial circumstances, which may or may not reflect the relative intrinsic activities under more normal conditions. Nevertheless, the mean value of $r 4_{b} /$ $r 1_{b}$ from the three reports is 0.9 , not different from 1 . Therefore, the calculations presented in Table 2 use $a=1$. Even if $a$ is as large as 2, and there are no data to point to its being larger the general conclusion is sustained that translocation alone is inadequate to account for a substantial portion, perhaps even most, of insulin-induced glucose uptake if the assumption is correct that only GLUTs 1 and 4 are involved.

Among the five reports summarized in Table 2, the concentration of GLUT4 in plasma membrane in response to insulin state would have to have been from $\sim 2$ to $\sim 7$ times greater than actually observed if translocation of GLUT to the plasma membrane were to account for the observed increase in glucose uptake. Calculations in Table 2 also show that if insulin had no effect on GLUT intrinsic activity, the fold increase in glucose uptake due to translocation alone would have ranged from 17 to $59 \%$ of that actually observed, with an average of about 35 to $40 \%$; that is, from the data in the five reports summarized in Table 2, insulin-induced increase in GLUT intrinsic activity accounts for more than half the observed increase in glucose uptake.

There is a comment in one report, which it is not necessary to cite in detail, that articulates what seems
GLUT4 were the only means by which insulin increased glucose uptake. $\%$ " $Q_{i}$ " $/ Q_{i}$, the percent of observed glucose uptake in the presence of insulin that can be accounted for only by translocation of GLUTs to the plasma membrane. ${ }^{\text {a }}$ This report gives two values for glucose uptake; the higher value (upper of the two rows) was obtained with $20 \mu \mathrm{mol} / 1$ glucose, the smaller value with $5 \mathrm{mmol} / \mathrm{l}$. ${ }^{\mathrm{b}}$ Plasma membrane preparations pooled from rat soleus and red and white gastrocnemius

to be a common misunderstanding. Some investigators hold that it is of no practical importance whether insulin increases GLUT intrinsic activity, because insulin increases plasma membrane GLUT4 by some 15-20 fold, at least in rat adipocytes, and even if insulin did increase GLUT intrinsic activity in those cells, it is probably only by a factor of about 2 . These investigators argue that a two fold increase is so small compared to a 15-20 fold that it must be of no practical consequence. This argument is irrelevant. It does not matter how large the insulin-induced fold increase in plasma membrane GLUT4 may be, or how small the fold increase in GLUT4 intrinsic activity. If the increase in plasma membrane GLUT4 density accounts for less than half the observed increase in glucose uptake and insulin-induced increase in GLUT intrinsic activity accounts for at least the other half, then the two insulin-induced processes play approximately equally important roles in producing insulin-induced glucose uptake. If insulin did not increase GLUT intrinsic activity, insulin-induced glucose uptake would only be about half or less of the glucose uptake actually observed.

\section{Are the data good enough for the analysis performed here?}

Before we discuss the analysis and its results, it is important to consider whether available data are suitable for this sort of analysis. In general, determination of glucose uptake and of insulin effect upon it are carried out under different conditions than those for quantitative determination of plasma membrane GLUT or their ratios with and without insulin. For example, in most cases glucose uptake is determined on a whole cell or population of cells, but plasma membrane GLUT densities have often been determined on preparations obtained by subcellular fractionation by density gradient centrifugation. Even when plasma membrane GLUT densities have been 
assessed by use of impermeant markers on the cell surface, there are more and different manipulations of the cells than for measurement of glucose uptake. An exception is the technique used in Horton's laboratory [21] in which both glucose uptake and GLUT densities are measured on preparations of rat skeletal muscle plasma membranes. A caution about Horton's method is that glucose uptake by the membrane preparation may underestimate whole muscle glucose uptake, as it has been reported to do, by a factor greater than 3 , in a comparison of glucose uptake in intact rat adipocytes glucose uptake in intact rat adipocytes with that in a plasma membrane fraction [30].

Prudence is also warranted in view of the small number of observations in much of the reported data. It is common for one category of experiment to have been carried out only three to five times; in one study, only once. Although this frugality may be justified by the technical complexity required, the numbers of observations in any one report may be too small, particularly in view of the great variations cited below, to justify confidence in the result.

There are also large differences in basal activity and in response to insulin among cell types (see Table 1), and between species, and with respect to age, all of which make it difficult to generalize. Some steps in assays of plasma membrane GLUTs may cause shift of GLUT. An example is translocation of skeletal muscle intracellular GLUT to the plasma membrane with hypoxia or muscle contraction [31, 32], both of which may occur during preparation of muscle for GLUT determination. Some artefact may be responsible for the wide variation in reported basal plasma membrane GLUT4 concentration. For example, in a meticulous study of 3T3-L1 adipocyte-like cells, Calderhead et al. [15] found a six-fold range among five experiments in basal GLUT4 cell surface density. There could even be wide variation between duplicates in the same experiment, especially when GLUT4 density was small; duplicates differed by as much as $100 \%$. Apparently there has been some uncontrolled variable.

Assessments of glucose transport and GLUT have been done under a variety of circumstances, that also handicap generalization. In some reports glucose uptake is measured over a range of glucose concentrations from moderately below levels at which clinical hypoglycaemia occurs to levels 10 times normal bas$\mathrm{al}$, in others glucose concentrations are less than $1 \%$ of normal basal (for the purpose of maintaining a negligible glucose concentration compared to purported equilibrium constant or Michaelis-Menten constant of GLUT-glucose reaction), and in some the ambient glucose concentration is normal basal.

If conclusions from the analysis of the data presented here turn out to be incorrect, the fault will be that presently available data on GLUT densities are artifactual, in the sense that conditions under which they were measured make the data inappropriate for comparison with glucose uptake. Despite the possibility that the database may prove to have been inappropriate, the data used for Tables 1 and 2 are what is available. The syntactical statements by which the data are analysed here will remain no matter what may or may not happen to the database; that is, it will be appropriate to analyse new data by the same set of syntactical statements as provided here.

If available data are not good enough for the analysis performed here, then they are also not good enough to justify any positive response to the question, does GLUT translocation account for the entire effect of insulin in stimulating glucose uptake?

\section{What if there are more than two GLUT isoforms in muscle and/or adipocytes?}

We concluded that one or both of the assumptions underlying our analysis of the data must be wrong because they led to inadmissible results. There were only two assumptions: (a) that no glucose is transported into any insulin-responsive cell except via either GLUT1 or GLUT4, and (b) that insulin has no effect on intrinsic activity of a GLUT to transport glucose. If there were one or more other GLUT isoforms present, would that invalidate the conclusion?

By steps similar to those that led to expression of eq(4), we arrive at a general expression for the case in which there are $n$ GLUT isoforms in the plasma membrane, where $n$ is any integer $\geq 1$ :

$\sum_{k=1}^{n}\left(\frac{Q_{i}}{Q_{b}}-\frac{\left(G T_{k}\right)_{i}}{\left(G T_{k}\right)_{b}}\right) r_{k}\left(G T_{k}\right)_{b}=0$

where we retain the assumption that insulin has no effect on any turnover number, so that each $r_{k i}=r_{k b} \equiv r_{k}$.

Analysis of the data in Tables 1 and 2 assures us (as far as available data can assure us) that if there is a GLUT isoform whose plasma membrane density in response to insulin increases by a greater fold than glucose uptake, it is not GLUT1 or GLUT4.

If insulin does not increase at least one turnover number there must be at least one term in eq(8) in which $\left(G T_{k}\right)_{i} /\left(G T_{k}\right)_{b}$ is greater than $\left(Q_{i} / Q_{b}\right)$ and sufficiently greater to compensate for all those terms in which $\left(Q_{i} / Q_{b}\right)>\left(G T_{k}\right)_{i} /\left(G T_{k}\right)_{\mathrm{b}}$. Eq $(8)$ points out that each factor $\left(Q_{i} / Q_{b}\right)-\left(G T_{k}\right)_{i}\left(G T_{k}\right)_{b}$ is multiplied by $r_{k}\left(G T_{k}\right)_{b}$. There is reason to take the family of $r \mathrm{~s}$ as not very different, based on published comparison of turnover numbers for GLUT1 and GLUT4. This implies that, if there is but a third GLUT isoform present, it must be abundant in the plasma membrane before exposure to insulin, as well as having a larger fold increase in plasma membrane density than the 
fold increase in glucose uptake. If this were so, it is likely, but not certain, that its presence would have been detected by now, as one has been in tissues other than those listed in Table 1. Palfreyman et al. [13] report that, on the basis of photolabelling plasma membrane GLUTs from 3T3-L1 adipocyte-like cells and immunoprecipitation of GLUTs 1 and 4, less than $20 \%$ of the photolabelled GLUT remained unprecipitated. Presumedly, this fraction represents some combination of unprecipitated GLUTs 1 and 4 and perhaps unidentified other isoform(s). Unfortunately, it is not stated whether this observation applies to conditions both before and in response to insulin. Assume that the less than $20 \%$ figure applies at least to basal conditions. Even if this entire fraction were a third GLUT isoform, and if this isoform were translocated to the plasma membrane in increasing numbers in response to insulin so that its fold increase in the plasma membrane exceeded the fold increase in glucose uptake, from the data of Palfreyman et al. [13] one can calculate that this unidentified GLUT would have to constitute more than $80 \%$ of plasma membrane GLUT in response to insulin to satisfy the requirement of eq (8) that there be no insulin effect on turnover number. On the other hand, if the unidentified GLUT were as much as $20 \%$ of plasma membrane GLUT in response to insulin and its fold increase exceeded that of glucose uptake and insulin had no effect on turnover number, it would have been less than $2 \%$ of basal GLUT, and its influence on eq (8) would have been negligible.

Although there are not yet sufficient data to be entirely confident, even if a third GLUT isoform is present it is likely to be at too small a density to affect the result of our analysis. From which we conclude, with only the reservation expressed in the previous sentence, that the inadmissible result demonstrates that insulin must have increased GLUT intrinsic activity.

\section{Previous reports of GLUT turnover numbers}

There have been three reports of efforts to find numerical values for GLUT1 and GLUT4 turnover numbers. There is only one report of an effort to determine effects of insulin on the isoform-specific turnover number, by Palfreyman et al. [13], adopting a model proposed by Calderhead et al. [15]. In accordance with an assumption commonly made before discovery of GLUT isoforms, Calderhead et al. [15] assumed that glucose transport via GLUTs 1 and 4 follows the steps required by the simplest MichaelisMenten model. This yielded a pair of equations containing eight unknowns. Calderhead et al. [15] wisely concluded that there were too many unknowns to yield to numerical evaluation.

Palfreyman et al. [13] hit upon ingenious methods to estimate numerical values for the eight unknowns: data analyses requiring combinations of graphic analysis of plots, intended to linearize, and searches for best least-squares fit. However, in most of the plots the data points appear to be not best fit by a straight line but by a monotonic curve, concave downward, perhaps asymptotic. On the other hand, a plot that ought not be linear, if the Michaelis-Menten model is correct, does seem to be linear. For those reasons, and because the final calculated value has had to be derived from a number of laboratory manoeuvers and a number of data analytical steps, and the analysis is model-dependent, it is not clear to what extent the calculated numerical values for the system parameters are reliable. Their analysis leads them to some surprising conclusions, in conflict with most other reports, for example, that GLUT1 became the major transporter in response to insulin, rather than GLUT4, which is reported in the overwhelming bulk of the literature to be the major insulin-responding GLUT.

From the data of Palfreyman et al. [13] we can calculate numerical values for the set of $r \mathrm{~s}$ at various glucose concentrations. Over a range of hypothetical glucose concentrations from 0.05 to $100 \mathrm{mmol} / 1 \mathrm{r} l_{i} /$ $r 1_{b}$ grows from 4 to 4.7 , while $r 4_{i} / r 4_{b}$ remains at about 1.4. Glucose uptake values, the family of $Q$ s, can be calculated from illustrated plots in Palfreyman et al. [13] and from tabulated values of the MichaelisMenten parameters. Ratios of $Q_{i} / Q_{b}$ vary from $\sim 15$ to $\sim 19$, depending largely on glucose concentration. In Table 2, calculation of the fraction of insulin-stimulated glucose uptake accounted for by enrichment of plasma membrane GLUT density used an average value for $a=r 4 / r 1=1$. This leads us to the estimate that in the experiments of Palfreyman et al. [13] translocation of GLUT to the plasma membrane accounted for $27 \%$ of insulin-stimulated glucose uptake, and increased GLUT intrinsic activity accounted for more than $71 \%$. If, instead, we set $a=1.69$, the value reported by Palfreyman et al. [13] for an equivalent ratio at maximum glucose concentration, GLUT translocation accounted for $32 \%$ of observed insulin-stimulated glucose uptake and increased intrinsic activity accounted for $68 \%$.

Unless the model-free analysis presented here is in error, its practical conclusion is that investigators ought to seek to understand the mechanism by which insulin increases GLUT intrinsic activity with as much vigor and ingenuity as they sought and seek mechanisms of translocation.

\section{References}

1. Cushman SW, Wardzala LJ (1980) Potential mechanism of insulin action on glucose transport in the isolated adipose cell. J Biol Chem 255: 4758-4762

2. Suzuki K, Kono T (1980) Evidence that insulin causes translocation of glucose transport activity to the plasma 
membrane from an intracellular storage site. Proc Natl Acad Sci USA 77: 2542-2545

3. Frost SC, Lane MD (1985) Evidence for the involvement of vicinyl sulfhydryl groups in insulin-activated hexose transport by 3T3-L1 adipocytes. J Biol Chem 260: 2646-2652

4. Blok J, Gibbs EM, Lienhard GE, Slot JW, Geuze HJ (1988) Insulin-induced translocation of glucose transporters from post-Golgi compartments to plasma membrane of 3T3-L1 adipocytes. J Cell Biol 106: 69-76

5. Calderhead DM, Lienhard GE (1988) Labeling of glucose transporters at the cell surface in 3T3-L1 adipocytes. Evidence for both translocation and a second mechanism in the insulin stimulation of transport. J Biol Chem 263: 12171-12174

6. Slot JW, Geuze HJ, Gigengack S, Lienhard GE, James DE (1991) Immuno-localization of insulin regulatable glucose transporter in brown adipose tissue of the rat. J Cell Biol 113: 123-135

7. Wardzala LJ, Jeanrenaud B (1981) Potential mechanism of insulin action on glucose transport in the isolated rat diaphragm. Apparent translocation of intracellular transport units to the plasma membrane. J Biol Chem 256: 7090-7093

8. Klip A, Ramlal T, Young DA, Holloszy JO (1987) Insulininduced translocation of glucose transporters in rat hindlimb muscles. FEBS Letters 224: 224-230

9. Hirschman MF, Goodyear LJ, Wardzala LJ, Horton ED, Horton ES (1990) Identification of an intracellular pool of glucose transporters from basal and insulin-stimulated rat skeletal muscle. J Biol Chem 265: 987-991

10. Wilson CM, Cushman SW (1994) Insulin stimulation of glucose transport activity in rat skeletal muscle: increase in cell surface GLUT4 as assessed by photolabeling. Biochem J 239: 755-759

11. Watanabe T, Smith MM, Robinson FW, Kono T (1984) Insulin action on glucose transport in cardiac muscle. $\mathrm{J}$ Biol Chem 259: 13117-13122

12. Uphues I, Kolter T, Goud B, Eckel J (1994) Insulin-induced translocation of the glucose transporter GLUT4 in cardiac muscle: studies on the role of small molecular weight proteins. Biochem J 301: 177-182

13. Palfreyman RW, Clark AE, Denton RM, Holman GD, Kozka IJ (1992) Kinetic resolution of the separate GLUT1 and GLUT4 glucose transporter activities in 3T3-L1 cells. Biochem J 284: 275-281

14. Clancy BM, Harrison SA, Buxton JM, Czech MP (1991) Protein synthesis inhibitors activate glucose transport without increasing membrane glucose transporters in 3T3-L1 adipocytes. J Biol Chem 266: 10122-10130

15. Calderhead DM, Kitagawa K, Tanner LI, Holman GD, Lienhard GE (1990) Insulin regulation of two glucose transporters in 3T3-L1 adipocytes. J Biol Chem 265: 13800-13808

16. Ezaki O, Fukuda N, Itakura H (1990) Role of two types of glucose transporters in enlarged adipocytes from aged obese rats. Diabetes 39: 1543-1549

17. Clark AE, Holman GD, Kozka IJ (1991) Determination of the rates of appearance and loss of glucose transporters at the cell surface of adipose cells. Biochem J 278: 235-241

18. Holman GO, Kozka IJ, Clark AE et al. (1990) Cell surface labeling of glucose transporter isoform GLUT4 by bisman- nose photolabel. Correlation with stimulation of glucose transport in adipose cells by insulin and phorbolester. $\mathbf{J}$ Biol Chem 265: 18172-18179

19. Murer E, Boden G, Gyda M, Deluca F (1992) Effects of oleate and insulin on glucose uptake, oxidation, and glucose transporter proteins in rat adipocytes. Diabetes 41 : 1063-1068

20. Goodyear LJ, Hirshman MF, Valyou PM, Horton ES (1992) Glucose transporter number, function, and subcellular distribution in skeletal muscle after exercise training. Diabetes 41: 1091-1099

21. Goodyear LJ, Hirshman MF, Smith RJ, Horton ES (1991) Glucose transporter number, activity, and isoform content in plasma membranes of red and white skeletal muscle. Am J Physiol 261: E556-E561

22. King PA, Horton ED, Hirschman MF, Horton ES (1992) Insulin resistance in obese Zucker rats (fa/fa) skeletal muscle is associated with a failure of glucose transporter translocation. J Clin Invest 90: 1568-1575

23. Brozinick JT jr, Elgen GJ jr, Yaspelkis BB III, Ivy IL (1994) The effects of muscle contraction and insulin on glucosetransporter translocation in rat skeletal muscle. Biochem $\mathbf{J}$ 297: 539-545

24. Zierath JR, He L, Guma A, Odegaard Wahlström E, Klip A, Wallberg-Henriksson H (1996) Insulin action on glucose transport and plasma membrane GLUT4 content in skeletal muscle from patients with NIDDM. Diabetologia 39: 1180-1189

25. Bruzinick JT jr, Yaspelkis BB III, Wilson CM, Grant KE, Gibbs M, Cushman SW, Ivy JL (1996) Glucose transport and GLUT4 protein distribution in skeletal muscle of GLUT4 transgenic mice. Biochem J 313: 133-140

26. Douen AG, Ramlal T, Cartee GD, Klip A (1990) Exercise modulates the insulin-induced translocation of glucose transporters in rat skeletal muscle. FEBS 261: 256-260

27. Lund S, Holman GD, Schmitz O, Pedersen O (1995) Contraction stimulates translocation of glucose transporter GLUT4 in skeletal muscle through a mechanism distinct from that of insulin. Proc Natl Acad Sci USA 92: 5817-5821

28. Nishimura H, Pallardo FV, Seidner GA, Vanucci S, Simpson IA, Birnbaum MJ (1993) Kinetics of GLUT1 and GLUT4 transporters expressed in Xenopus oocytes. J Biol Chem 268: 8514-8520

29. Dauterive R, Laroux S, Bunn RC, Chaisson A, Sanson T, Reed BC (1996) C-terminal mutations that alter the turnover number for 3- $\mathrm{O}$-methylglucose transport by GLUT1 and GLUT4. J Biol Chem 271: 11414-11421

30. Armoni M, Harel C, Burvik R, Karnieli E (1995) Modulation of the activity of glucose transporters (GLUT) in the aged/obese rat adipocyte: suppressed function, but enhanced intrinsic activity of GLUT. Endocrinology 136: 3292-3298

31. Douen AG, Ramlal T, Klip A, Young DA, Carter GD, Holloszy JO (1989) Exercise induced increase in glucose transporters in plasma membranes of rat skeletal muscle. Endocrinology 124: 449-454

32. Carter GD, Douen AG, Ramlal T, Klip A, Holloszy JO (1991) Stimulation of glucose transport in skeletal muscle by hypoxia. J Appl Physiol 70: 1593-1600 